\title{
The Design and Mathematical Modelling of Novel Extensor Bending Pneumatic Artificial Muscles (EBPAMs) for Soft Exoskeletons
}

\author{
Hassanin Al-Fahaam*, Steve Davis and Samia Nefti-Meziani \\ Autonomous Systems and Robotics research centre, School of Computing/Science and Engineering, The University of Salford, Manchester, \\ United Kingdom.
}

\begin{abstract}
This article presents the development of a power augmentation and rehabilitation exoskeleton based on a novel actuator. The proposed soft actuators are extensor bending pneumatic artificial muscles. This type of soft actuator is derived from extending McKibben artificial muscles by reinforcing one side to prevent extension. This research has experimentally assessed the performance of this new actuator and an output force mathematical model for it has been developed. This new mathematical model based on the geometrical parameters of the extensor bending pneumatic artificial muscle determines the output force as a function of the input pressure. This model is examined experimentally for different actuator sizes. After promising initial experimental results, further model enhancements were made to improve the model of the proposed actuator. To demonstrate the new bending actuators a power augmentation and rehabilitation soft glove has been developed. This soft hand exoskeleton is able to fit any adult hand size without the need for any mechanical system changes or calibration. EMG signals from the human hand have been monitored to prove the performance of this new design of soft exoskeleton. This power augmentation and rehabilitation wearable robot has been shown to reduce the amount of muscles effort needed to perform a number of simple grasps.
\end{abstract}

Keywords: soft robotics, soft mechanism, wearable robot, artificial pneumatic rubber muscle, modelling.

\section{Introduction}

Exoskeleton robots are a blend of human intelligence and machine power [1]. Typically, this means the force output of the human wearer is improved by the device. During recent decades researchers across the world have been seeking to improve this technology [2]. This has resulted in recent practical advances in the fields of mechanical design, electronic engineering, biomedical design and computerized reasoning. Exoskeleton robots are anticipated to play a critical part in the fields of rehabilitation, assistive mechanical technology and enhancing human performance [3-7].

Many exoskeleton robots have been created for different purposes with their own particular benefits and faults. An exoskeleton moves with the wearer and applies power to their joints to give them increased strength and stamina. They can also provide power assistance to elderly people who may have muscle weakness or struggle to control their limbs. These systems can also be used in rehabilitation to improve the ability of a disabled patient to control their limbs efficiently and independently.

Power augmentation and rehabilitation wearable robots (exoskeletons) must be: i) inherently safe, because they are in direct contact with humans, ii) lightweight, for easy use and portability, and iii), able to fit a person without extensive adjustment and calibration.

Unfortunately, traditional actuators and rigid, serial link manipulators are not well suited to these requirements. However, the relatively new fields of soft robotics have the potential to address these issues.

* Corresponding author.

E-mail address: h.s.h.al-fahaam@edu.salford.ac.uk (Hassanin Al-Fahaam). 
During the last two decades, power assist and/or rehabilitation robots have been attracting more interest [8]. Polygerinos, et al. [9] designed a power assisted glove for full finger assistance of the activities of daily living. The glove is manufactured from elastomeric and inextensible materials to be lightweight and to create soft actuators that conform to the patient's fingers and are able to apply enough force to the hand to create a grasp. A fluidic pressure controller dependent on a human users intentions is used to actuate and control the glove. Patient intention is detected by monitoring the EMG signals from electrodes placed on the forearm. Polygerinos, et al. [10] presented a soft glove to assist the rehabilitation exercises for functional grasp pathologies. The prototype employs soft actuators consisting of moulded elastomeric chambers with fibre reinforcements that produce suitable curvatures at finger joints under fluid pressurization. A closedloop controller is used to regulate the pressure inside the actuators. Another design of power assistive wearable glove was developed by Noritsugu, et al. [11] using two joint muscles for each finger except the thumb. Two curved actuators with different diameters are connected in series on the back of each finger, and the muscle with the smaller diameter is placed on terminal two joint of the finger and the bigger diameter one on the root joint, with each muscle provided by a split air supply. The advantage of this technique is the ability to control the finger joints separately and to provide more movement types for assistance and rehabilitation. Similar to this, a design with two joints for each finger has been demonstrated by Toya, et al. [12]; but this device used soft materials moulded to create the bending type artificial muscles. The controller of this design depends on human intention as the input by using bending sensors attached to the muscles. In addition, the movements are divided into three modes (power grip, precision grip, and tip pinch) and the controller predicts the mode by matching the grasping angles with a stored database. A further design of hand power assistance wearable robot was developed by Kadowaki, et al. [13]. The design of this wearable robot is completely made of soft materials and features bending pneumatic muscle like actuators. Each muscle has two bladders, a rubber one and the other one a balloon covered by woven elastic. These muscles are placed on the back of the hand, one for each finger, except that the thumb has two muscles. PI controllers were used to decide the bending angles for the fingers, based on a bending sensor attached with each finger joint. Estimated timing is used to perform the release movement after the gripping movement. They tried to use the EMG signals to control the release movements, but they faced a problem due to the similarity between the EMG signals from the fingers and the wrist joint movements. Improvement of the controller algorithm of this robot has been achieved by [14]. A neural network controller is used (Self-Organizing Maps) to recognise the different EMG signals from the wrist and the fingers at release movement; but this method assumed the fingers and the wrist move independently (if the fingers are moving, the wrist is fixed, and conversely).

The 3D printed pneumatic soft actuators have also been used in rehabilitation devices such as the hand rehabilitation wearable glove proposed by Polygerinos, et al. [15]. The new soft actuators are formed from elastomeric materials and feature a network of pneumatic channels which create bending movements that are applied to the patient's fingers. The bending curvature and force response of these actuators was explored utilizing a finite element model (FEM) and geometrical analysis before they were assessed practically. Another 3D printed glove was presented by Yap, et al. [16]; this exoskeleton has variable stiffness features to perform rehabilitation movements. The main drawback of this research is that they developed different actuators to provide each rehabilitation movement meaning the patient needed to replace the gloves actuators when converting to a different exercise mode.

One of the main problem of using soft actuators is in the mathematical modelling which is highly complex because of their nonlinear behaviour.

\subsection{Pneumatic Muscles}

One actuator which is potentially of use in the development of soft exoskeleton systems is the Pneumatic Artificial Muscle (PAM). Pneumatic artificial muscles vary from conventional pneumatic actuators and in this way can be utilized as driving components of portable, bionic, anthropomorphic and humanoid robots; physiotherapeutic and rehabilitation robots and also for the mechanization of industrial processes. Pneumatic muscle actuators can be considered as single-acting pneumatic actuators [17].

One of the major drivers for researchers to develop and use pneumatic muscles is the performance similarity between them and organic muscles [18-20]. Tsagarakis and Caldwell [21] analysed these similarities as illustrated in Table 1. The most common pneumatic muscle design is based on the McKibben muscle. Pneumatic artificial muscles have been used in many biologically inspired robots as well as in soft robots such as continuum robots [22]. As pneumatic muscles are constructed from soft materials, they have the capability to provide much safer interaction with humans than is possible with traditional rigid robots.

A pneumatic muscle consists of an internal elastomeric bladder surrounded by a woven braided shell. As the actuator is pressurised it experiences a liner change in length. Depending upon how the muscle is constructed it will either contract when pressurised (contractor muscle) or extend (extensor muscle). No single muscle is capable of both types of motion and contractor muscles have seen considerably more research than extensor 
muscles. As they produce linear contractions when pneumatic muscles are used to produce exoskeletons they must either be combined with a rigid serial link mechanism [19] or the actuators must be secured rigidly to the user [23]. Both of these approaches mean the systems cannot easily be adjusted for different users and the use of rigid structure reduce inherent safety.

Table 1. Comparison of pneumatic artificial muscles and natural muscles.

\begin{tabular}{|c|c|c|}
\hline Parameter & $\begin{array}{c}\text { Organic } \\
\text { Muscle }\end{array}$ & PAM \\
\hline Displacement & $35 \%$ & $30-68 \%$ \\
\hline Force/cm ${ }^{2}$ & $20-40 \mathrm{~N}$ & $100-500 \mathrm{~N}$ \\
\hline Power/Weight & $40-250 \mathrm{~W} / \mathrm{Kg}$ & $500-2000 \mathrm{~W} / \mathrm{kg}$ \\
\hline Efficiency & $45-70 \%$ & $32-50 \%$ \\
\hline Control & Good & Fair-Good \\
\hline Operation in Water & Yes & Yes \\
\hline Temperature Range & $0-40^{\circ} \mathrm{C}$ & $-30-80^{\circ} \mathrm{C}$ \\
\hline Robustness & Excellent & Fair-Good \\
\hline Energy Source & Chemical & Pneumatic \\
\hline Environment Safe & Produces $\mathrm{CO}_{2}$ & Yes \\
\hline Scalable from & $\mu \mathrm{m}-\mathrm{m}$ & $\mathrm{cm}-\mathrm{m}$ \\
\hline
\end{tabular}

This paper proposes a novel form of pneumatic muscle which bends when activated. It is suggested that this actuator can be incorporated into a soft exoskeleton suit which envelopes the user like an item of clothing. When activated the suit will deform applying forces to the user's limbs causing their joints to flex. The paper first describes the construction of the new extensor bending pneumatic artificial muscle. A number of prototypes are developed and their behaviour assessed experimentally. A new mathematical model of the actuator is then developed and verified against experimental results. Unlike the bending actuator presented by Kadowaki, et al. [13] the novel bending muscle presented in this work is based on the McKibben muscle. Unlike Kadowaki's muscle the muscle used in this work converts radial pressure on the inside of the entire muscle into bending force giving greater force capacity. The muscle used in this work can also be used to a higher pressure than that reported by Kadowaki further increasing the force that can be generated. The new actuator is then demonstrated in first prototype of an exoskeleton glove for hand force augmentation and rehabilitation. The performance of this exoskeleton is examined experimentally.

\section{Modelling Pneumatic Muscles}

In addition to developing the novel bending pneumatic muscle; this paper attempts to model its behaviour. This required a detailed review of attempts model the behaviour of traditional extensor and contractor pneumatic muscles.

There are numerous modelling types for contracting PAMs [24]. However, there is currently no $100 \%$ accurate mathematical model for these actuators, because of their highly nonlinear behaviour, and the materials they are constructed from (latex or rubber) also show high hysteresis behaviour. Most previous models have concentrated on contracting PAMs and there is a lack of models for extensor PAMs and bending muscles [25]. Many methods have been used to create efficient controllers for these soft pneumatic actuators, but this effort is hindered by the lack of accurate mathematical models.

There have been numerous applications of PAMs demonstrated over the last 20 or so years covering many fields such as biorobotic, medical, industrial, and aerospace applications [26].

Recently, there has been a considerable amount of research into the mathematical modelling of contraction PAMs. The purpose of modelling approaches is to create a mathematical relationship between the length of the PAM, the amount of pressure inside it, and the force it generates. These mathematical models depend on variable parameters such as applied pressure, axial force, length and diameter of the PAMs, as well as the properties of materials used. All these variables play a considerable role in the dynamic behaviour of the soft actuators. There is strong evidence of the non-linear behaviour of PAMs; thus, the major challenge is to build a robust controller for this $[27,28]$. A classification of the most common and valuable mathematical models was made by [25]. Below are some types of mathematical modelling of contraction PAM.

\subsection{Geometrical model of contraction PAM}

The basic mathematical modelling approach was based on the geometric characteristics of PAMs. The models of Chou \& Hannaford [29] and Tondu \& Lopez [30] have been widely used. Chou and Hannaford's 
model was based on static features of the soft actuator; they assumed that the muscle is cylindrical in shape, ignored the extensibility of the threads in the sleeve, ignored the friction force between the sleeve and the bladder and between the threads of the sleeve, and ignored rubber bladder forces. Their model depends on the geometry parameters of the actuator such as the length of the actuator, the length of the strands used to form the braid, the number of times the strand circles the muscle and the angle between braid strands. Chou and Hannaford improved their model by adding a new parameter representing the sleeve and bladder thickness to reduce the error, but the error between the mathematical model and the experimental results was still 15-20\%. According to Paynter [31], a stretch of the actuator braided material is caused as a result of increasing muscle volume when input pressure is applied on fixed muscle length. Davis et al. [32] proved Paynter's theory by practical experiments; the results of these experiments showed around 5\% increase in the length of the thread across the test range, and this increase depended on the applied pressure and the muscle length. The extension of the braid must be calculated to derive a valid mathematical model. Based on Doumit, Fahim, and Munro [33], more realistic geometric measurements have been added to Chou and Hannaford's models. Chou and Hannaford assumed that the PAM is perfectly cylindrical, but actually there is a deformation at both ends of the muscle. Doumit et al.[33] assumed the deformation to be conical in shape at both ends and the middle part is a cylinder.

\subsection{Contraction PAMs' phenomenological model}

The axial output force of a PAM depends on the contraction ratio. Serres, Reynolds, Phillips, Rogers, and Repperger [34] suggested that this behaviour is similar to a combination of a spring, a damper, and a contractile elements placed together in parallel. These elements each had a mathematical model, and then they derived a new phenomenological PAM model depending on these models. Experiment verification was conducted and this model worked efficiently with an acceptable amount of error between the mathematical model and the experimental results.

\subsection{Empirical model of contraction PAM}

There is a functional similarity between a PAM and a mechanical spring because both produce a tensile force when attached to a load. For the spring force is produced by its material used to form it and for a PAM the force is a function of applied pressure. Based on this idea, Wickramatunge and Leephakpreeda [35] proposed a PAM model based on adding a new parameter to the mechanical spring model; this parameter is the gauge pressure inside the artificial muscle.

\subsection{Curved contraction PAM model}

There are numerous mathematical models for ordinary linear PAMs but there are no models for bending muscles like those proposed in this paper. However, Zhang, Yang, Chen, Zhang, and Dong [36] proposed two approximate models based on physics: the membrane and beam models for pneumatic muscles operating when curved around an object. These two models were used to control the joint torque of a wearable elbow exoskeleton.

\section{Extensor Bending Pneumatic Artificial Muscles (EBPAM)}

The proposed extensor bending pneumatic artificial muscles (EBPAMs) are based on linearly extending McKibben artificial muscles. These muscles are reinforced along one side keeping one side of the actuator at a fixed length. This means that when pressurised the new actuator does not extend in length but rather bends.

Development of the bending pneumatic muscle began with the experimental analysis of a series of extending pneumatic muscles. A prototype pneumatic muscle (M1) was produced which consisted of a rubber bladder with resting length $160 \mathrm{~mm}$ and diameter $10 \mathrm{~mm}$. This was encased by a braided nylon sleeve whose length was double that of the rubber and had a minimum diameter of $8 \mathrm{~mm}$ and maximum diameter of $18 \mathrm{~mm}$. The muscle had two 3D printed terminals at either end, one closed and the other containing a small hole for the supplying of pressurised air. The rubber and braid were secured to the terminals using cable ties and the resulting actuator had an experimentally measured resting diameter of $18 \mathrm{~mm}$; the same of maximum diameter of the sleeve.

Fig. 1 shows the behaviour of the unloaded muscle under different supplied pressures. Due to the fact the braided sleeve was significantly longer than the rubber tube (meaning the muscle had a resting braid angle greater than $54.7^{\circ}$ ) an increase in the supplied pressure causes an increase in the muscle length i.e. the muscle is of the extending type. The length of muscle increases with increasing supplied pressure; the maximum increase in length was measured to be $68 \%$.

Fig. 2 illustrates the characteristic behaviour of extending pneumatic muscle relative to the supplied pressure. During operation, an axial compressive force is produced at the end of the actuator.

By reinforcing one side of the extending pneumatic muscle using a fixed length thread with a $500 \mathrm{~N}$ breaking strength, as shown in Fig. 3, the muscle is converted to a bending actuator. The thread prevents one side of the 
actuator from extending whilst the opposite remains free to extend when pressurised. The resultant difference in length of the two sides of the actuator mean it bends as seen in the figure.

Fig. 3 shows the configuration of the EBPAM at four arbitrary but increasing pressures. It can be seen that one side of the actuator remains at a fixed length whilst the over side increases in length leading to bending of the actuator.

The bending angle of the proposed actuator increases with increasing supplied pressure and this relationship was explored experimentally. The pressure in the actuator was gradually increased and the angle of the remote end of the muscle relative to its initial position was measured. Fig. 4 illustrates the relationship between the supplied pressure and the bending angle of the proposed artificial muscle.

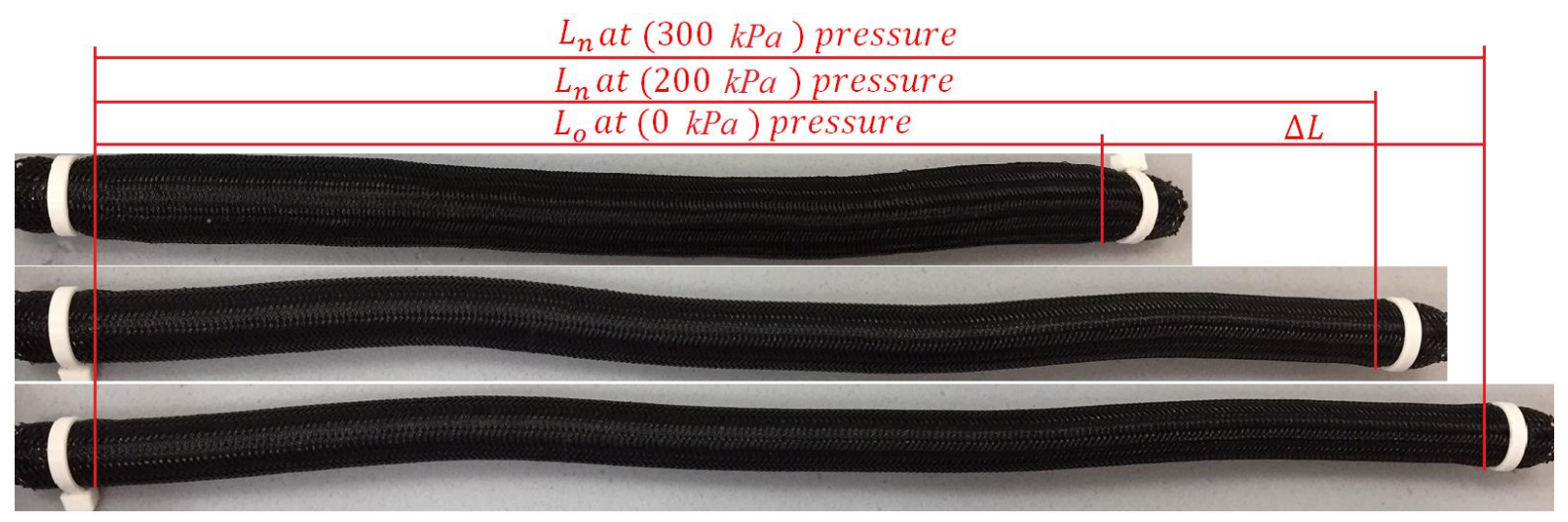

Fig. 1. EPAM with different pressures, where: $L_{o}$ is the muscle length without pressurization, $L_{n}$ is the muscle length under pressure and $\Delta L$ is the increase in length.

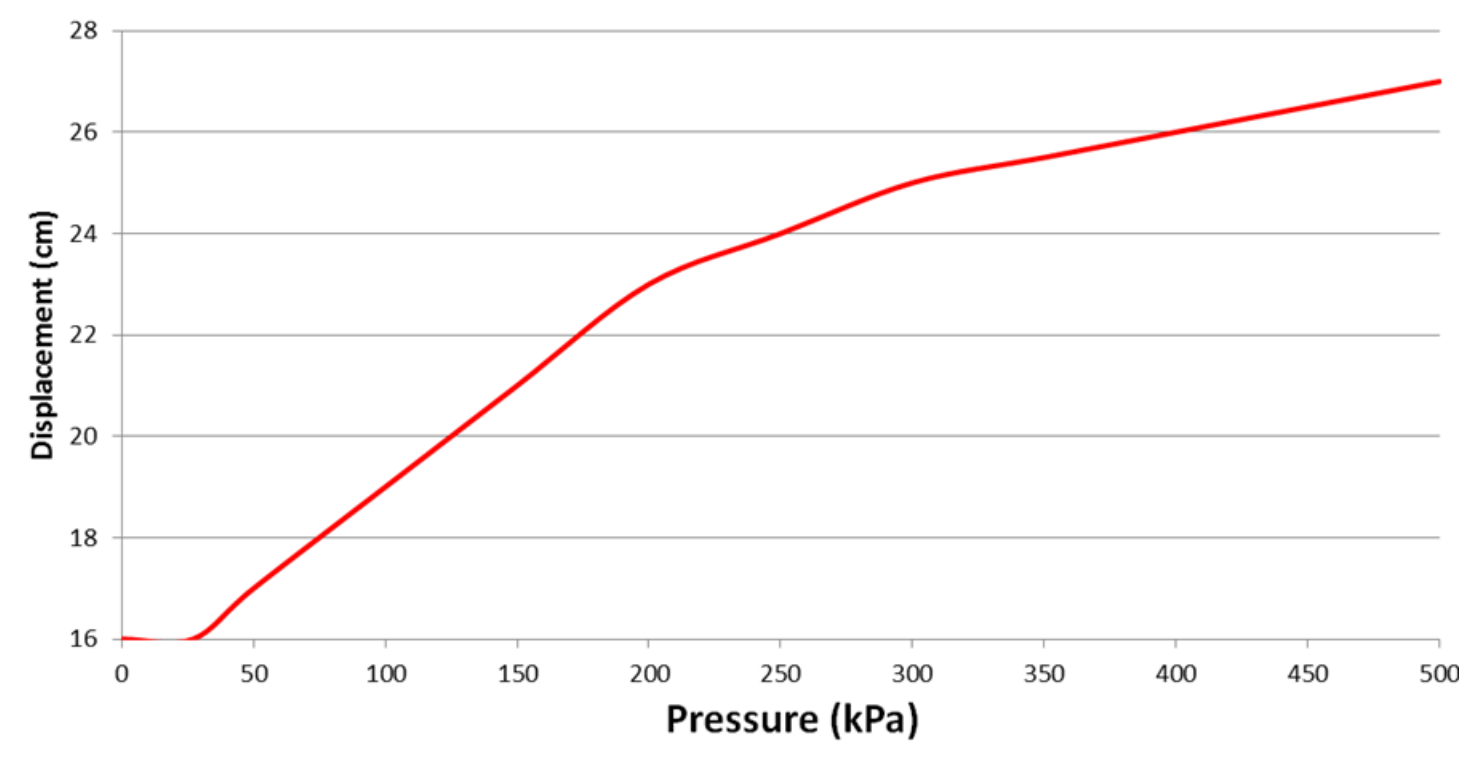

Fig. 2. EPAM length related to the supplied pressure. 


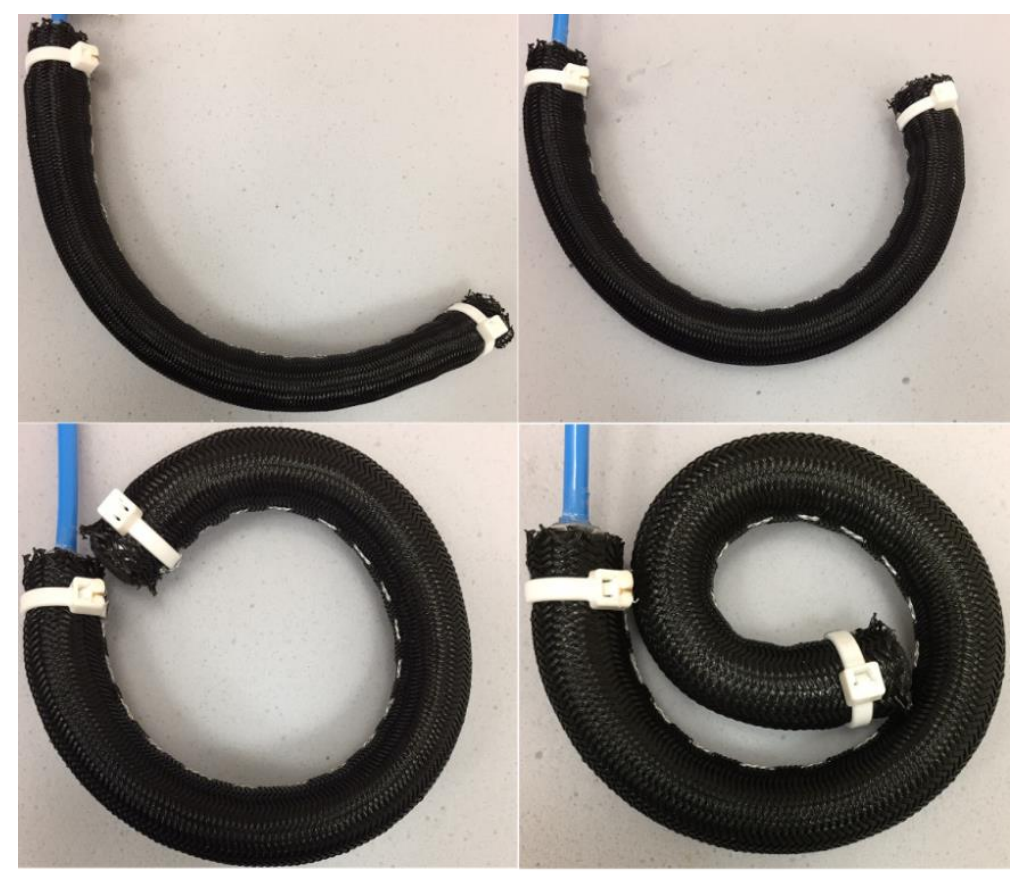

Fig. 3. EBPAM pressurised by different amounts of pressure.

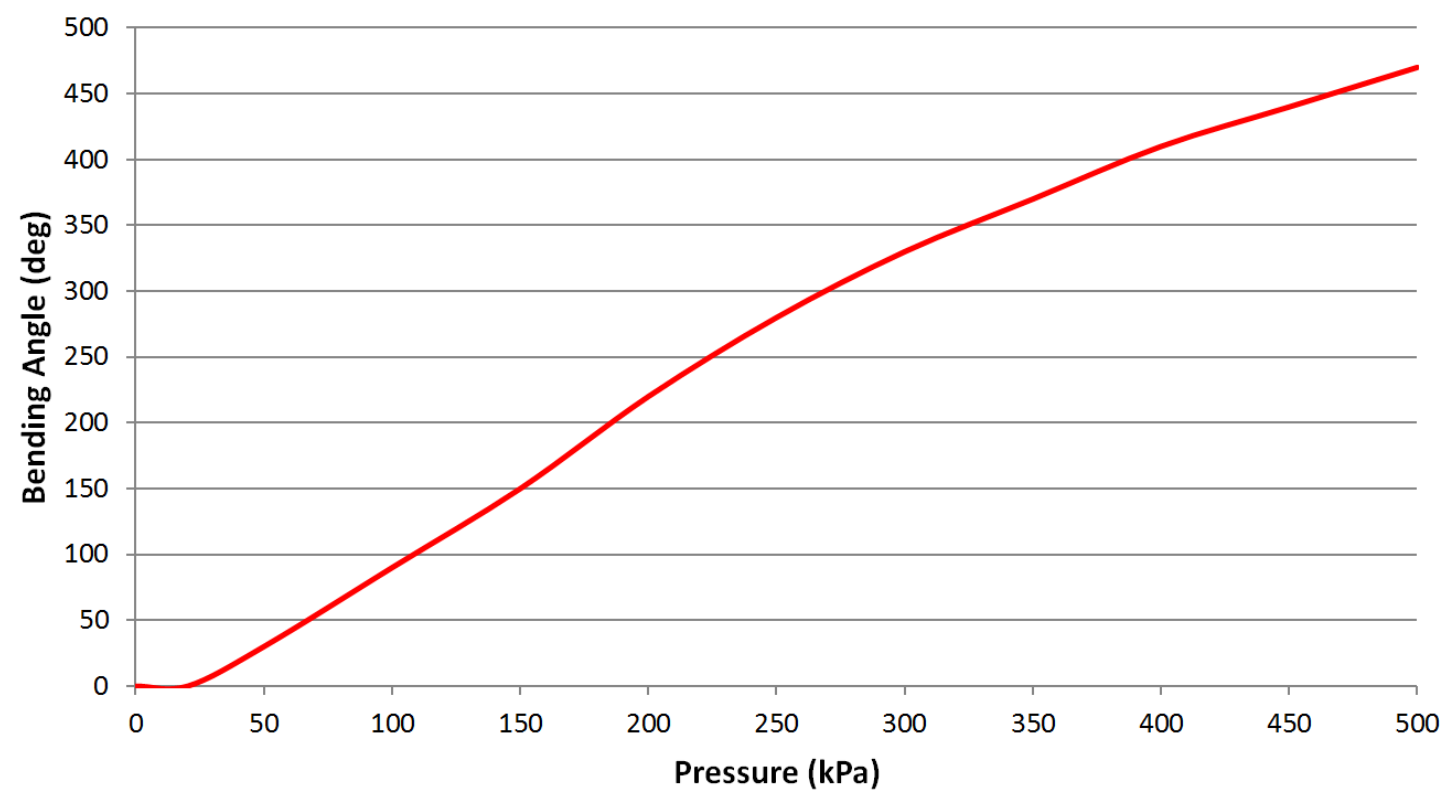

Fig. 4. EBPAM bending angle with the supplied pressure.

\subsection{Experimental Investigation of Extensor Bending Pneumatic Artificial Muscles (EBPAM)}

To verify these finding two further EBPAMs, M2 and M3 were created which used the same end caps and bladder as the first muscle (M1) but used larger diameter braid, as can be seen in Table 2. Larger diameter braid results in higher muscle force, so each muscle prototype generates a greater force that the previous.

Table 2. M1, M2 and M3 characteristics.

\begin{tabular}{|l|}
\hline Muscle No. \\
\hline Muscle length \\
\hline Bladder diameter \\
\hline Sleeve diameter \\
\hline Muscle diameter \\
\hline
\end{tabular}

\begin{tabular}{|l|l|c|}
\multicolumn{1}{c|}{ M1 } & \multicolumn{1}{c|}{ M2 } & \multicolumn{1}{c|}{ M3 } \\
\hline $160(\mathrm{~mm})$ & $160(\mathrm{~mm})$ & $160(\mathrm{~mm})$ \\
\hline $10(\mathrm{~mm})$ & $10(\mathrm{~mm})$ & $10(\mathrm{~mm})$ \\
\hline $8(\mathrm{~mm})$ & $10(\mathrm{~mm})$ & $12(\mathrm{~mm})$ \\
\hline $18(\mathrm{~mm})$ & $23(\mathrm{~mm})$ & $35(\mathrm{~mm})$ \\
\hline
\end{tabular}


Fig. 5 (a) and (b) illustrates the relationship between the applied pressure and the actuator length for muscle M2 and M3 respectively before the reinforcing thread is added. It can be seen that in both instances the extending ratio is $68 \%$ which is the same as for M1. This is not an unexpected result as it is the braid length, not its diameter, which determines the muscles maximum extending. Fig. 5 (c) and (d) shows the relationship between the pressure and bending angle for M2 and M3 respectively. The relationship is broadly the same for all three muscle. Again and for the same reason, this is not an unexpected result. However, at high pressure, it can be seen that the results for the three muscle diverge. The reason for this can be seen in Fig. 5 (e) and (f), as the muscle approaches maximum bending it collides with itself and the muscle becomes deformed. As can be seen, this problem is more significant for the larger diameter muscles.

This section has experimentally proven the operation of the EBPAM; the following sections will attempt to analyse its behaviour mathematically and develop a numerical model.

\section{Kinematic Analysis of the Proposed EBPAM}

The general geometry of a McKibben muscles is shown in Fig. 6. The middle section of the muscle is perfectly cylindrical and the muscle has length $L$, diameter $D$ and an angle between a single braided thread and the actuator's central axis $\theta$. The braid is formed from many individual strands of entire length $b$ which encircle the actuator $n$ times.

An extensor muscles differs from a contracting muscle in that the resting length of the braid is significantly longer than the length of the bladder. This means the braid must be compressed (braid angle increased) to make it the same length as the bladder. This means that the muscle will have an unpressurised braid angle greater than $54.7^{\circ}$ (the minimum energy configuration), when pressurised the braid angle will reduce in an attempt to reach the minimum energy state and this will result in extension of the muscle. The bending muscle is derived from the extensor one by reinforcing one side of the braid. This means that one side of the braid $\theta$ is always at its maximum value and cannot increase in length. When pressurise the increase in actuator length will only occur on the free side and this results in bending.
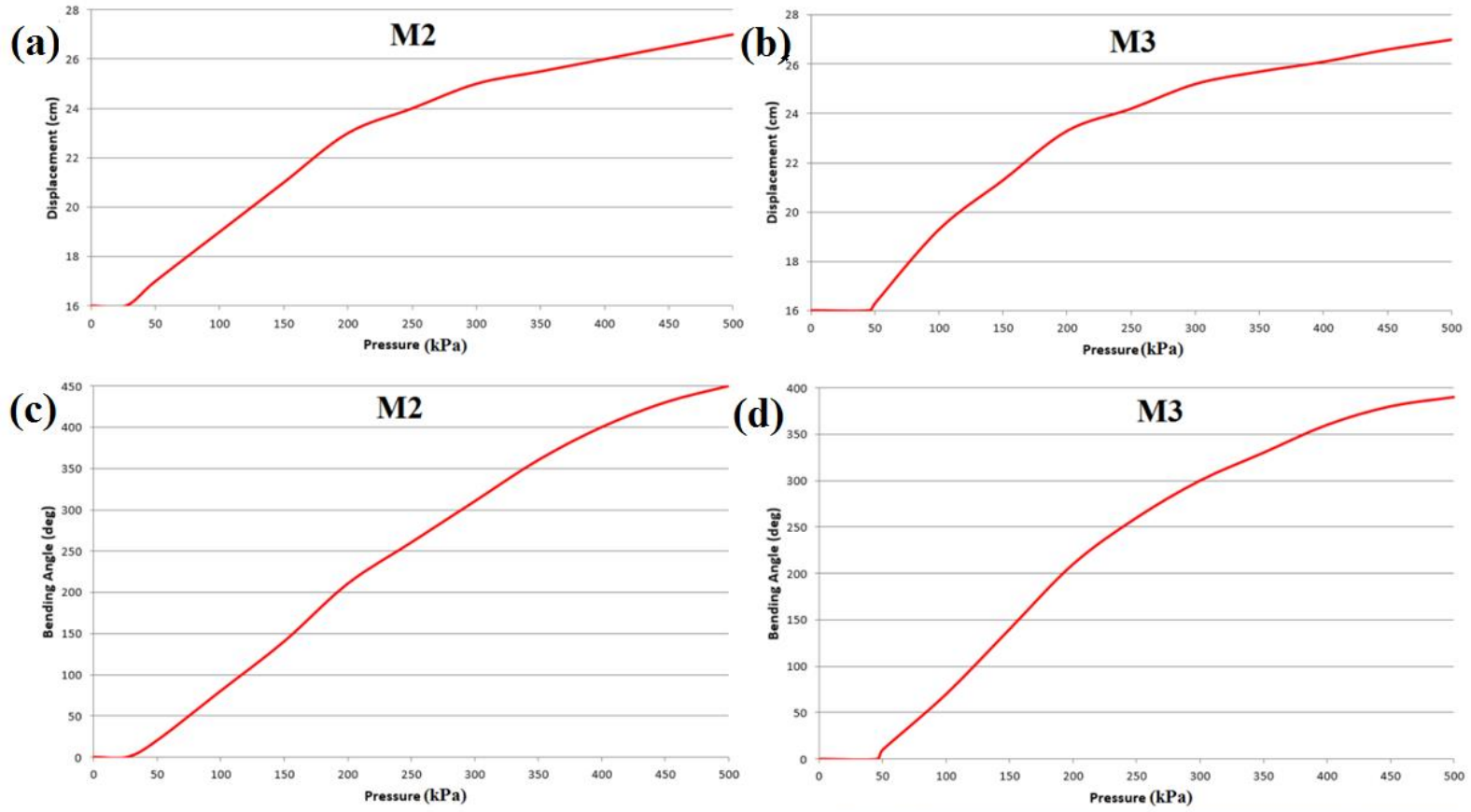

(e)

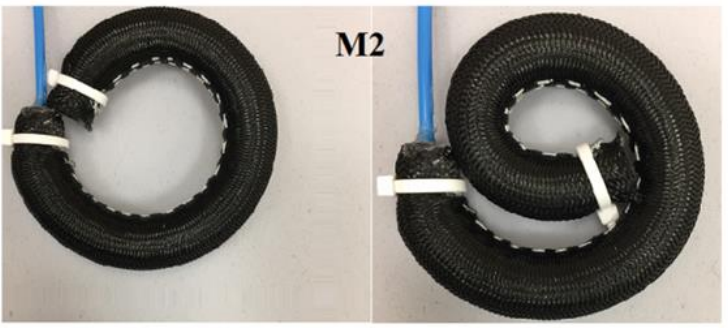

(f)

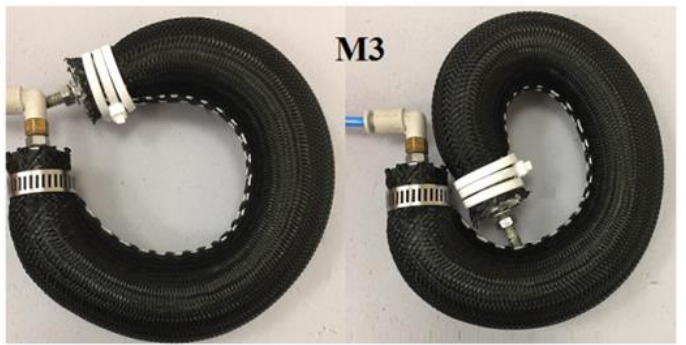

Fig. 5. M2 and M3 with their characteristics. 

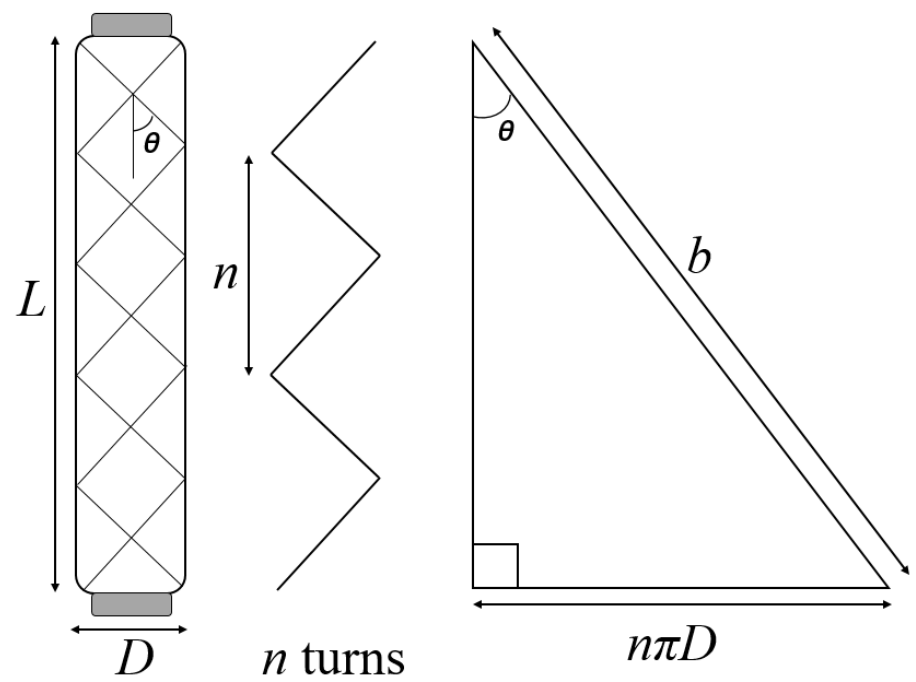

Fig. 6. The general geometry of McKibben muscles.

From Fig. 6 the initial length of the actuator will be:

$$
L=b \cos \theta
$$

And the muscle diameter:

$$
D=\frac{b \sin \theta}{n \pi}
$$

The analysis of the EBPAM is based on the following assumption: the muscle retains a circular cross-section during bending, the threads used to form the sleeve and reinforce one side of the muscle are inextensible, there is no friction force between the sleeve and the bladder and between the threads of the sleeve and there are not elastic forces within the bladder.

Fig. 7 shows the bending muscle geometry, where $L_{o}$ is the muscle length on the reinforced side, $L_{n}$ is the length of bending muscle on the free side, $D_{c}$ is the curved muscle diameter, $\alpha$ is the bend angle of the muscle, $r_{o}$ is the inner radius and $r_{n}$ is the outer radius. The actuator length will be the average length:

$$
L_{c}=\frac{L_{o}+L_{n}}{2}
$$

The actuator diameter in relation to the inner and outer radius is given by:

$$
D_{c}=r_{n}-r_{o}
$$

Combining (1) with the equation for the length of an arc the following equations can be produced which describing the length the two opposite sides of the bending muscle:

$$
\begin{gathered}
L_{o}=b \cos \theta_{\max }=r_{o} \alpha \\
L_{n}=b \cos \theta=r_{n} \alpha
\end{gathered}
$$

The radius inside the curve is determined by the maximum braid angle $\left(\theta_{\max }\right)$, which is fixed as a result of the reinforcing thread, and the radius outside the curve is determined by the braid angle $\theta$, which reduces as the muscle bends. 


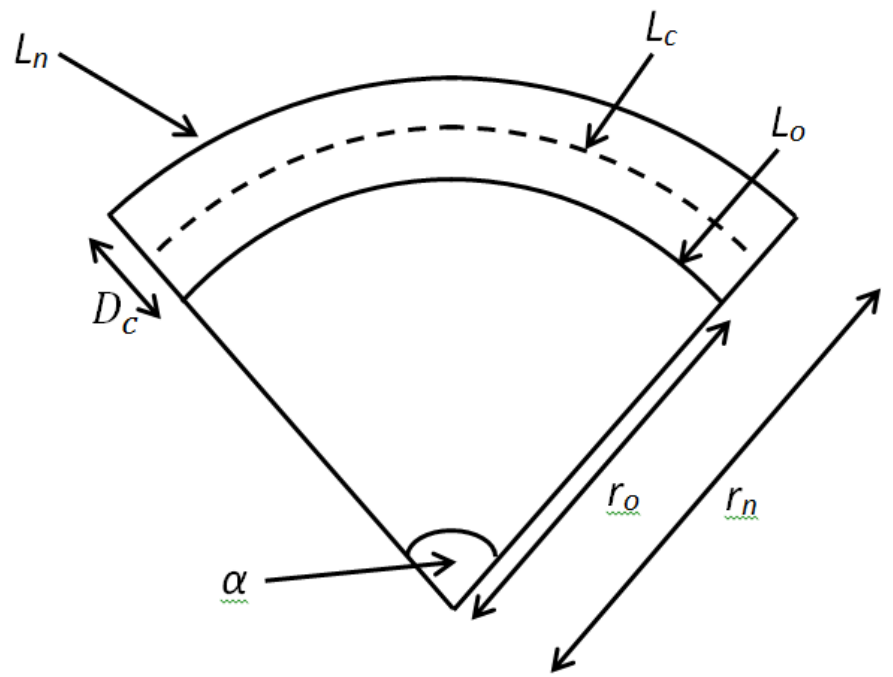

Fig. 7. Bending muscle geometry.

Unlike a traditional pneumatic muscle, the braid angle around the circumference of the bending muscle is not a constant. Instead the braid angle will decrease around the circumference from $\theta$ on the outside edge of the bend (point A in Fig. 8) to $\theta_{\max }$ at on the inner edge of the bend (point B in Fig. 8). Each of these braid angles will have an associated muscle diameter as shown in Fig. 8. If we assume that the muscle cross section forms a perfect circle, then the overall diameter will be the sum of the radiuses on the outside of the curve $\left(r_{I}\right)$ and inside of the curve $\left(r_{2}\right)$ as shown in Fig. 8. Equation (2) can be used to determine the diameter of the bent muscle:

$$
\begin{gathered}
r_{1}=\frac{D_{1}}{2}=\frac{b \sin \theta}{2 n \pi} \\
r_{2}=\frac{D_{2}}{2}=\frac{b \sin \theta_{\text {max }}}{2 n \pi} \\
D_{c}=r_{1}+r_{2} \\
D_{c}=\frac{b \sin \theta+b \sin \theta_{\text {max }}}{2 n \pi}
\end{gathered}
$$

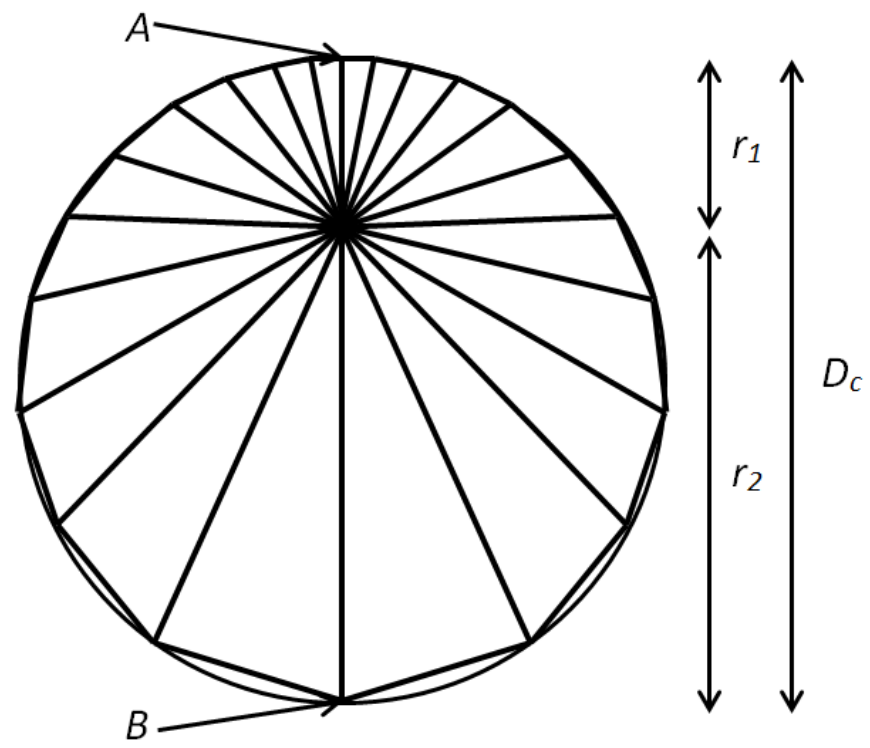

Fig. 8. Radii inside curved muscle. 
Using the above information, it is possible to develop the kinematic equations of the bending muscle which describe the bending angle $\alpha$, the radius of curvature $r_{o}$ and the length of the central axis of the muscle $L_{c}$ as follows:

By substituting Equation (4) in Equation (6):

$$
L_{n}=\left(D_{c}+r_{o}\right) \alpha
$$

Substituting Equations (5) and (6) in Equation (11) gives the following equation:

$$
L_{n}=\left(D_{c}+\frac{L_{o}}{\alpha}\right) \alpha=D_{c} \alpha+L_{o}=b \cos \theta
$$

From Equations (12) and (5) we can derive the curvature angle as a function of $\theta$ and $\theta_{\max }$ using the following equation:

$$
\alpha=\frac{b \cos \theta-b \cos \theta_{\max }}{D_{c}}
$$

Also, the actuator length can be determining by substituting Equations (5) and (6) in Equation (3):

$$
L_{c}=\frac{b \cos \theta_{\max }+b \cos \theta}{2}
$$

\section{Modelling the Output Force of the Proposed EBPAM}

The previous section presented a kinematic analysis of the bending muscle. However, if the bending muscle is to be used in an application, it is important that its force generating behaviour is also understood. This analysis is performed using the theory of conservation of energy as was performed by Chou et al. [29] for a contracting pneumatic muscle.

The input work $W_{\text {in }}$ which occurs in the artificial muscle is in the form of applied air pressure which act on the inner surface of the actuator and leads to a change in actuator volume. This can be described by the following formula:

$$
d W_{i n}=\int_{s_{i}}\left(P^{\prime}-P_{o}\right) d l_{i} \cdot d_{s_{i}}=\left(P^{\prime}-P_{o}\right) \int_{s_{i}} d l_{i} \cdot d_{s_{i}}=P \cdot d V_{c}
$$

Where $P^{\prime}$ is the internal absolute air pressure, $P_{o}$ is the environment pressure $(103360 \mathrm{~Pa}$ on the day of testing), $P$ is the relative differential air pressure, $S_{i}$ is the actuator inner surface, $d l_{i}$ is the inner surface displacement vector, $d_{s_{i}}$ is the area vector, and $d V_{c}$ is the actuator volume change.

Based on the volume of a cylinder volume formula:

$$
V=\frac{\pi D^{2} L}{4}
$$

Therefore the curved actuator volume $V_{c}$ will be:

$$
V_{c}=\frac{\pi D_{c}{ }^{2} L_{c}}{4} \rightarrow V_{c}=\frac{b^{3}\left(\cos \theta+\cos \theta_{\max }\right)\left(\sin ^{2} \theta+2 \sin \theta_{\max } \sin \theta+\sin ^{2} \theta_{\max }\right)}{32 \pi n^{2}}
$$

Where $D_{c}$ is the curved muscle diameter from Equation (10) and $L_{c}$ is the central curved muscle length from Equation (14).

The output work $W_{\text {out }}$ done when the EBPAM bends is associated with an increase in actuator length $L_{c}$ as a result of the change in volume. This can be described by the following formula:

$$
d W_{\text {out }}=F \cdot d L_{c}
$$

Fig. 9 shows the EBPAM output force direction. 


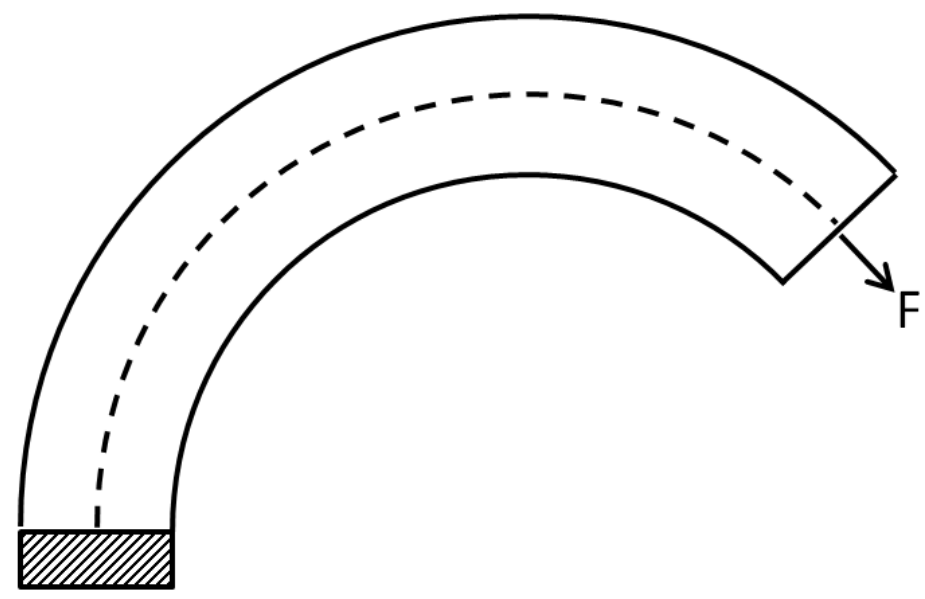

Fig. 9. EBPAM output force direction.

From the theory of energy conservation the change in input work is equal to the change in output work:

$$
d W_{\text {in }}=d W_{\text {out }}
$$

The output force can therefore be determined by combining (15) an (18) as follows:

$$
F=P \frac{d V_{c}}{d L_{c}}
$$

Differentiating with respect to $\theta$ gives:

$$
\frac{d V_{c}}{d \theta}=\frac{b^{3}}{32 \pi n^{2}}\left(\begin{array}{c}
\left(\cos \theta+\cos \theta_{\max }\right)\left(2 \cos \theta \sin \theta+2 \sin \theta_{\max } \cos \theta\right) \\
-\sin \theta\left(\sin \theta+\sin \theta_{\max }\right)^{2}
\end{array}\right)
$$

and:

$$
\frac{d L_{c}}{d \theta}=\frac{-b \sin \theta}{2}
$$

The final mathematical model of the proposed EBPAM output force results from substituting Equations (21) and (22) in Equation (20) as follows:

$$
F=\frac{P b^{2}}{8 \pi n^{2}}\left(\begin{array}{c}
\frac{\left(\cos \theta+\cos \theta_{\max }\right)\left(\cos \theta \sin \theta+\sin \theta_{\max } \cos \theta\right)}{\sin \theta} \\
-\frac{\left(\sin \theta+\sin \theta_{\max }\right)^{2}}{2}
\end{array}\right)
$$

Equation (23) relies values of $\theta$ which is particularly difficult to measure directly with a high degree of accuracy. However, equation (14) allows $\theta$ to be determined from the measured muscle length. As the length of the muscle is considerably easier to measure, in the experimental verification described in the next section the muscle length is measured and then $\theta$ calculated using (14).

\subsection{Experimental Verification of Model}

To validate this output force mathematical model of the bending muscles the three muscles (M1, M2 and M3), previously described, were again used. These muscles were each placed in a rig, which held them in an isometric configuration at a range of known bending angles, as can be seen in Fig. 10. A load cell was mounted to the remote end of the muscle to allow actuator force to be measured and recorded to a PC. 


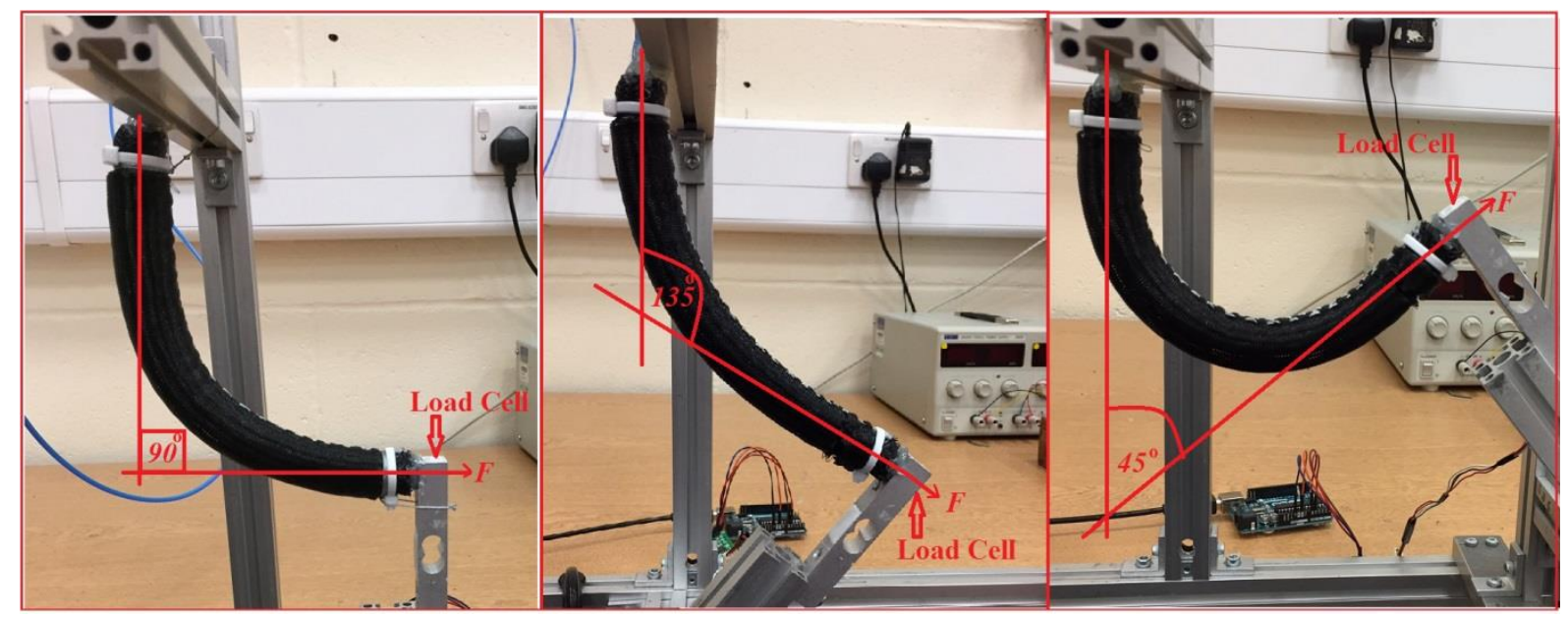

Fig. 10. Test rig used to measure muscle force at a range of bending angles.

Each muscle to be tested was placed in the rig and then the pressure applied to it was increased from 50kPa to $500 \mathrm{kPa}$ in $50 \mathrm{kPa}$ increments and the force generated was recorded. The experiment was repeated at muscle bend angles of $90^{\circ}, 135^{\circ}$ and $45^{\circ}$. The experimental results along with the modelled data can be seen in Fig. 11.
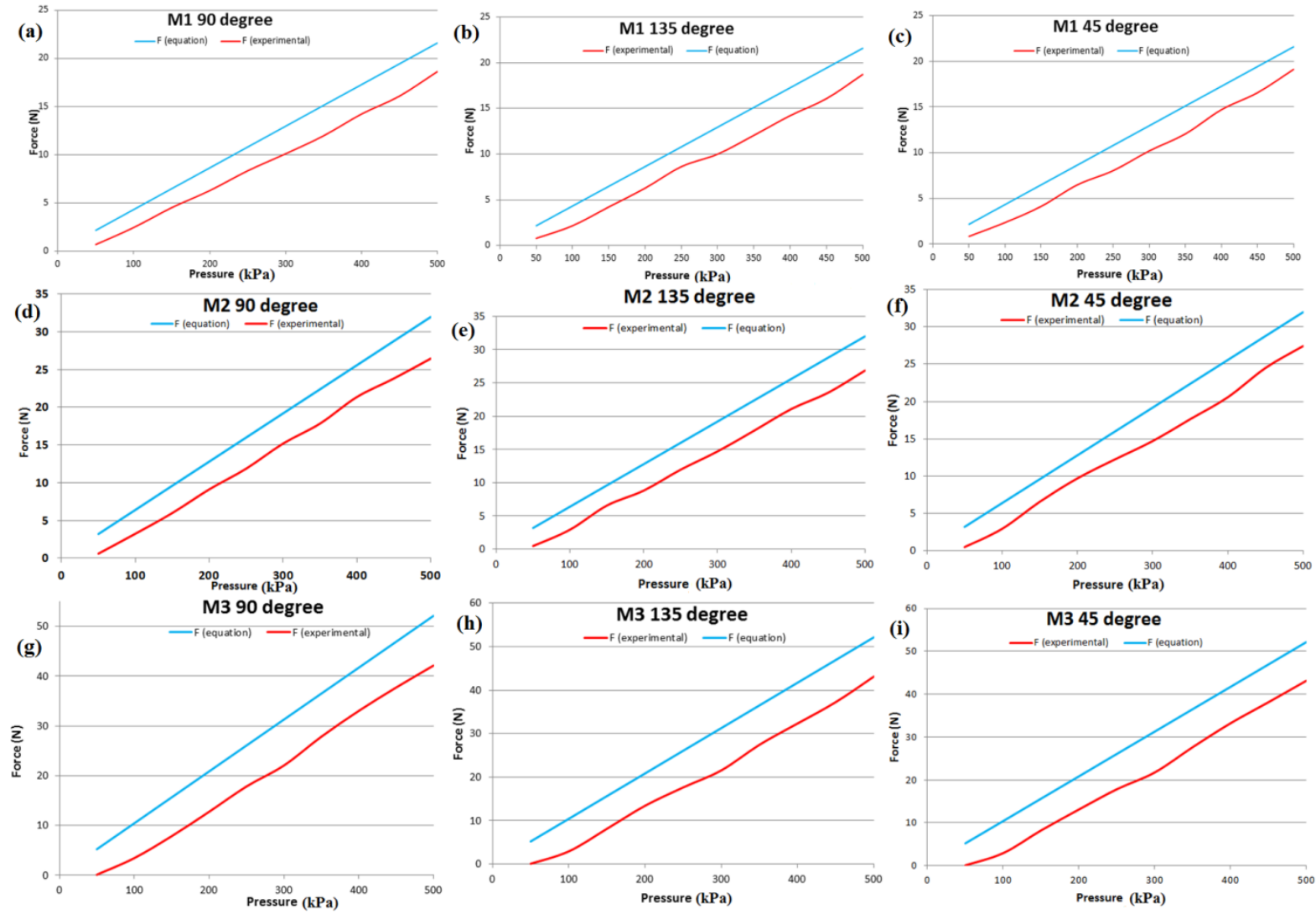

Fig. 11. The measured and modelled force output for muscles M1, M2 and M3 at $90^{\circ}, 135^{\circ}$ and $45^{\circ}$ bend angle.

There is an obvious observable error between the experimental and theoretical results in the form of a relatively constant over estimation of each muscle's force output. For M1 the average error across all three bend angles was calculated to be $2.65 \mathrm{~N}$. This was determined by measuring the force error at each pressure value for each of the three bend angles and then taking an average of these values. This represents an error of $13.36 \%$ of the maximum force generated by the muscle. For M2 and M3 the average errors were $4.02 \mathrm{~N}$ and $8.19 \mathrm{~N}$ respectively. These values correspond to $14.95 \%$ and $19.13 \%$ of the maximum output force for the two muscles M2 and M3. 
These errors are not unexpected as the model used is simplistic and does not consider energy losses within the muscle. However, the accuracy of the simple model is broadly similar to when the same approach has been used to model contracting pneumatic muscles [32].

\section{Enhancements to the Mathematical Model}

From the previous section, it is clear that there is a considerable gap between the theoretical and experimental results. There are many factors which introduce energy losses in PAMs. One of these factors is the energy spend radially expanding the rubber bladder before it makes contact with the braided sleeve.

Fig. 12 shows there is a certain amount of pressure needed to inflate the bladder tube until it comes into contact with the sleeve, and below this pressure the actuator output force will be zero [21, 37].

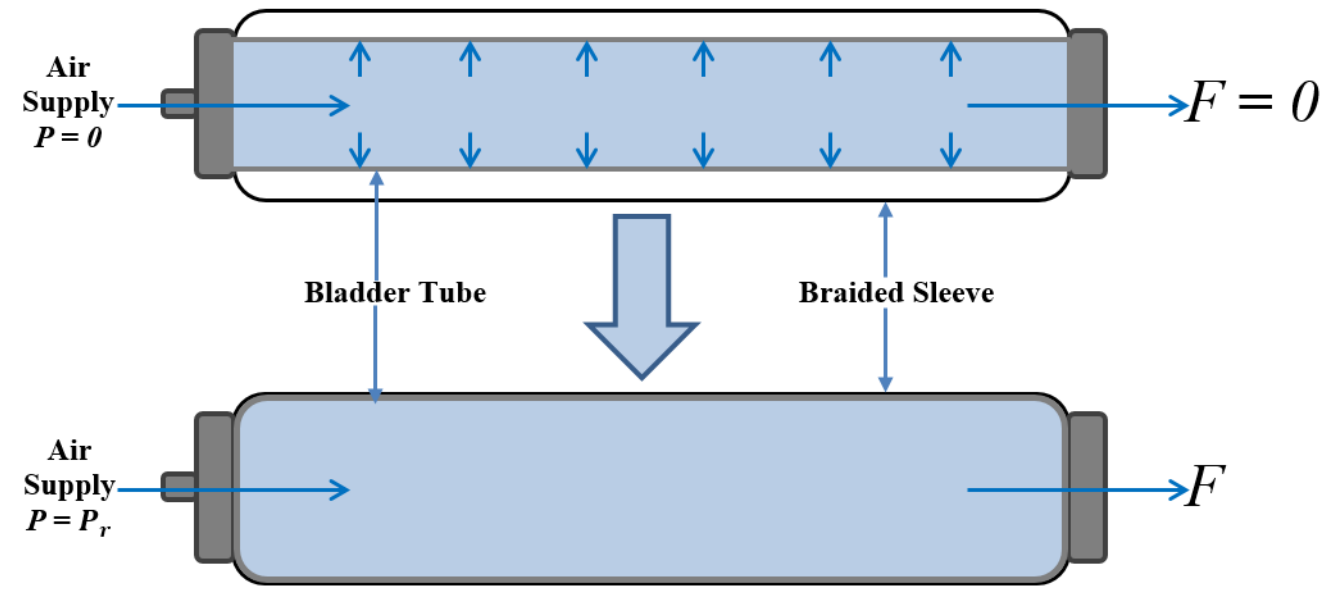

Fig. 12. Pressure needed to inflate the bladder tube.

We will call this pressure needed to radially expand the bladder to make contact with the braid $P_{r}$. To take into consideration this lost pressure effect, the active drive pressure for the actuator in the model (Equation 23) is replaced with the actual pressure $P_{a}$ where:

$$
P_{a}=P-P_{r}
$$

Fig. 13 shows the experimental results obtained for $P_{r}$ for the three test muscles M1, M2 and M3. The results were obtained by pressurising the bladders and measuring their diameter as the pressure increased up to the point where the diameter was equal to that of the resting braid diameter.

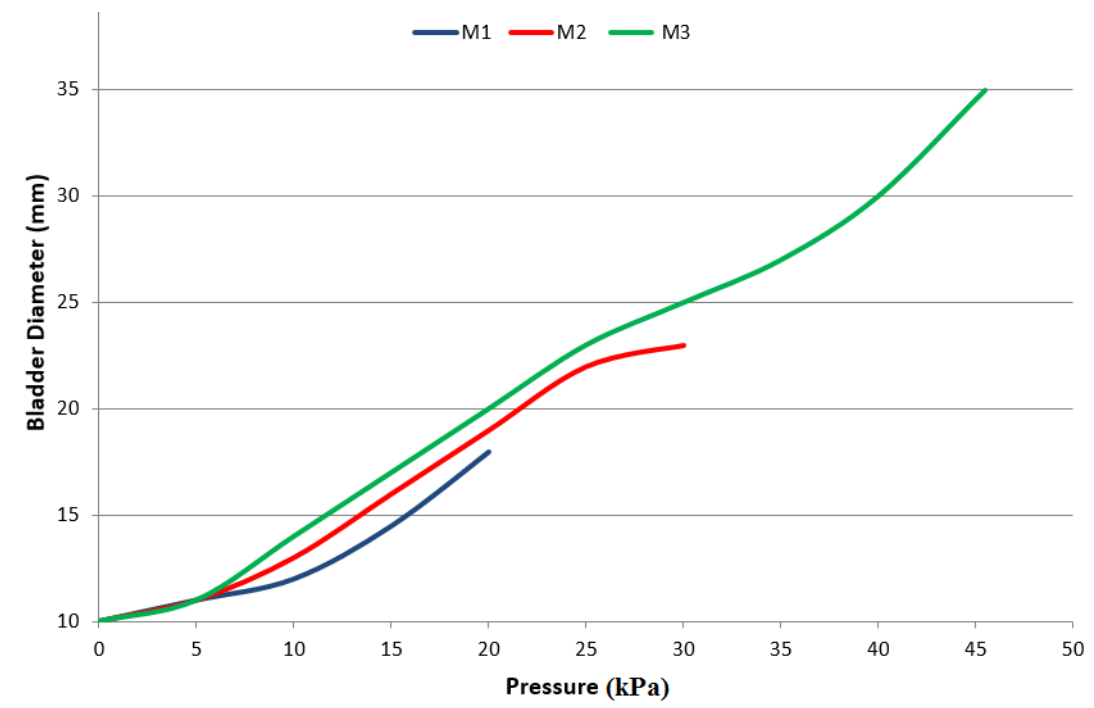

Fig. 13. Pressure needed to inflate rubber. 
Tsagarakis and Caldwell [21] modelled the radial expansion pressure for their contraction muscle. Based on this model, we derived a new model of this lost pressure experimentally.

We represent the radial expansion pressure $P_{r}$ in the following equation:

$$
P_{r}=K_{r}\left(D_{o}-D_{c} \sin \theta\right)
$$

Where $D_{o}$ is the actual bending muscle diameter and $K_{r}$ is the linearized radial actuator elasticity obtained from the experiment above [21]:

$$
\begin{gathered}
K_{r}=2000 \mathrm{kPa} / \mathrm{m} \text { for } D_{c} \sin \theta<\frac{D_{o}}{2} \\
K_{r}=500 \frac{\mathrm{kPa}}{\mathrm{m}} \text { for } D_{c} \sin \theta>\frac{D_{o}}{2}
\end{gathered}
$$

The final output force model of extensor bending muscles is given by the following equation:

$$
F=\frac{P_{a} b^{2}}{8 \pi n^{2}}\left(\begin{array}{c}
\frac{\left(\sin \theta+\sin \theta_{\max }\right)^{2}}{2} \\
-\frac{\left(\cos \theta+\cos \theta_{\max }\right)\left(\cos \theta \sin \theta+\sin \theta_{\max } \cos \theta\right)}{\sin \theta}
\end{array}\right)
$$

\subsection{Experimental Verification of Enhanced Model}

Fig. 14 shown the performance of the new model when compared to the experimental and previously modelled results.
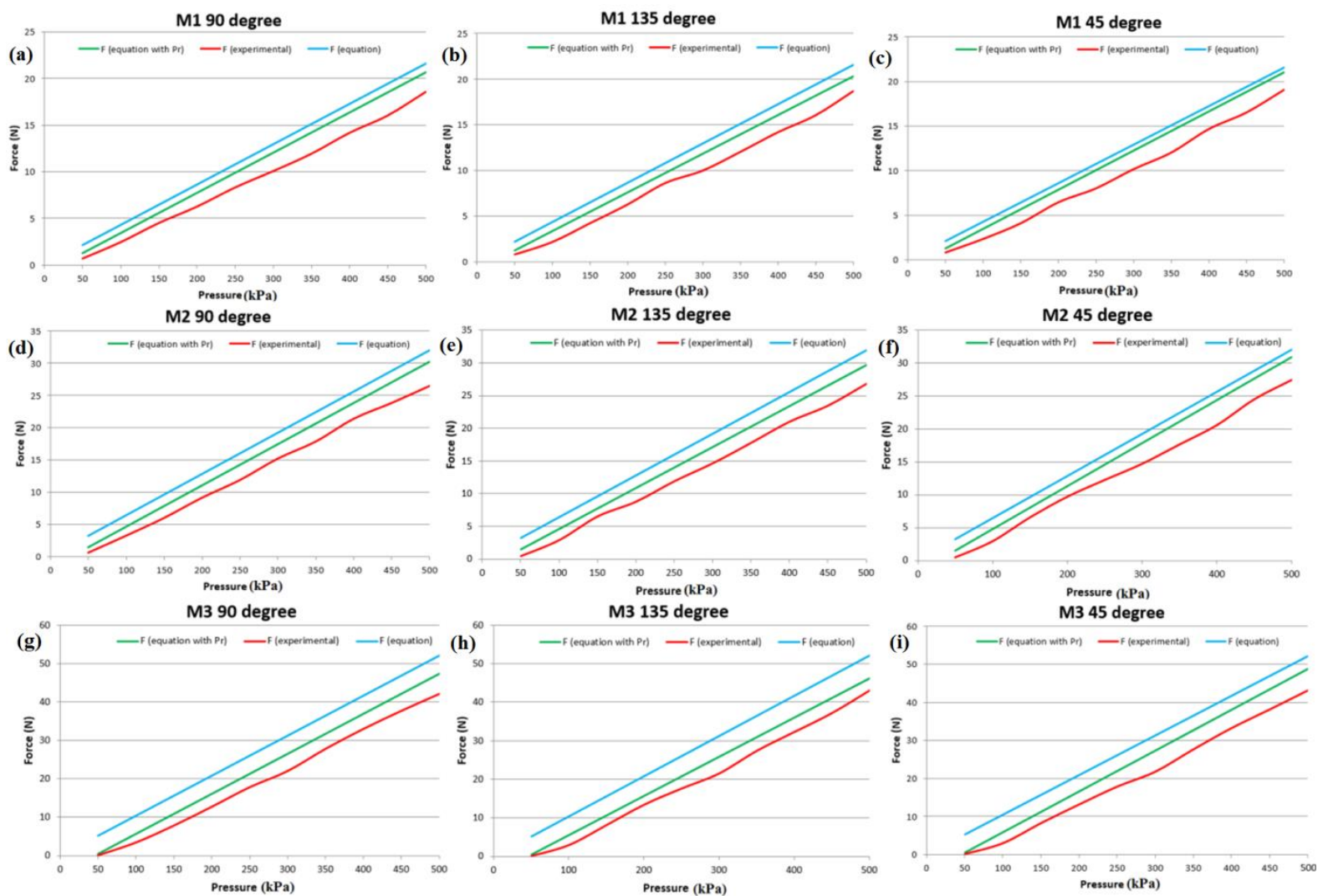

Fig. 14. Final results of the output force for M1, M2 and M3.

It is clear from the graphs in Fig. 14 that the average percentage errors are decreased by a considerable amount when using the new model. Table 3 illustrates a comparison between the previous errors and the new errors for all proposed bending muscles at bending angles of $90^{\circ}, 135^{\circ}$ and $45^{\circ}$. 
Across the three bending angles the average percentage error for muscles M1, M2 and M3 were found to be $8.62 \%, 8.59 \%$ and $8.11 \%$ respectively. The percentage reduction in modelled error when $P_{r}$ was included was $35.48 \%, 42.54 \%$ and $57.61 \%$ for M1, M2 and M3 respectively. It is obvious, this enhancement stage reduces the error of high muscles diameters more than the muscles with small diameters because of we used the same bladder diameter for all muscles, then the muscle with bigger sleeve maximum diameter has the maximum lost of radial expansion pressure.

Table 3. The average error as a percentage of the maximum actuator force with and without consideration of $P_{r}$.

\begin{tabular}{|c|c|c|c|c|c|c|}
\hline Muscle No. & \multicolumn{2}{|r|}{ M1 } & \multicolumn{2}{|r|}{ M2 } & \multicolumn{2}{|r|}{ M3 } \\
\hline Bending Angle & $\%$ Error & \% Error with $\mathrm{P}_{\mathrm{r}}$ & $\%$ Error & $\%$ Error with $\mathrm{P}_{\mathrm{r}}$ & $\%$ Error & $\%$ Error with $\mathrm{P}_{\mathrm{r}}$ \\
\hline $90^{\circ}$ & 13.70 & 8.98 & 15.29 & 8.79 & 19.47 & 8.20 \\
\hline $135^{\circ}$ & 13.68 & 7.88 & 15.32 & 7.77 & 19.21 & 6.75 \\
\hline $45^{\circ}$ & 12.71 & 9.01 & 14.25 & 9.21 & 18.71 & 9.37 \\
\hline
\end{tabular}

\section{Soft Glove}

The application for the new bending extensor pneumatic muscle is in a soft power assist glove for force augmentation and rehabilitation. The main requirements of the glove are:

- Inherent safety - as it is in direct contact with a human user.

- Lightweight - to allow portability.

- Fast response - actuation speed must match that of the human user.

- High force to weight ratio - to keep weight low.

- Low/no calibration requirement - so system, can easily be used by multiple people.

One of the disadvantages of traditional exoskeletons is that they have discrete joints which must be correctly aligned with the user's joints $[11,16,38]$. Incorrect alignment can lead to injury and so adjustment and calibration is required when a different person uses the system. The main benefits of the bending muscle is that is does not have discrete joints, instead the entire actuator flexes. If the actuator is mounted to the back of a finger, when pressurised it will apply force to the entire rear of the finger resulting in bending at the joints. Based on this concept a soft force augmentation glove has been produced. The bending muscles are sewn onto the reverse of a leather glove as shown in Fig. 15.

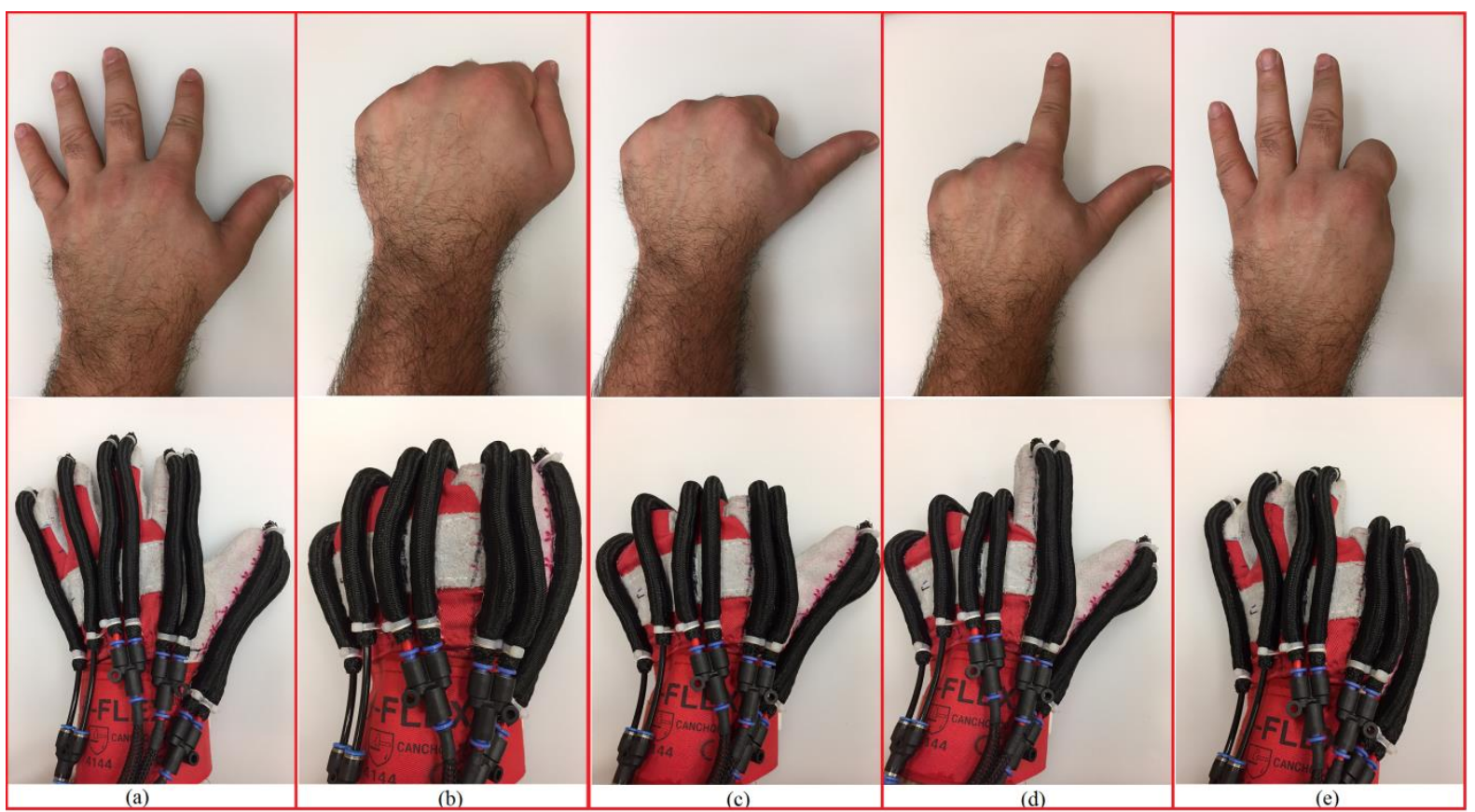

Fig. 15. The proposed soft glove with some rehabilitation movements

Eight muscles are used, two muscles for the thumb, index and middle finger to increase force and single muscles on the ring and little fingers. The resultant soft glove has a weight of approximately $0.15 \mathrm{Kg}$. The air 
supply to all the muscles is controlled by MATRIX 3-3 solenoid valves which are pulse width modulated to allow control of the airflow. In the experiment described below the glove is controlled in an open loop manner.

In order to demonstrate the effectiveness of the glove in simple force augmentation applications two grasping experiments were conducted both with and without the use of the glove. The experiments involved a user grasping a $0.5 \mathrm{~kg}$ mass using a pinch grasp and a $1 \mathrm{~kg}$ mass using a whole hand grasp.

During experimentation, the human test subject was fitted with EMG (Electromyography) sensors on their forearm. These were used to record the total muscle activity required to perform the grasping tasks. The results can be seen in Fig. 16(a) and 17(a) for the two grasps when the glove was not used. The subject then repeated the two tasks whilst using the glove. For the pinch grasp test the thumb and index finger actuators were pressurised to $300 \mathrm{kPa}$ and for the full hand grasp, all actuators were pressurised to $300 \mathrm{kPa}$. The user's muscle activity was again recorded using EMG and the results can be seen in Fig. 16(b) and 17(b).

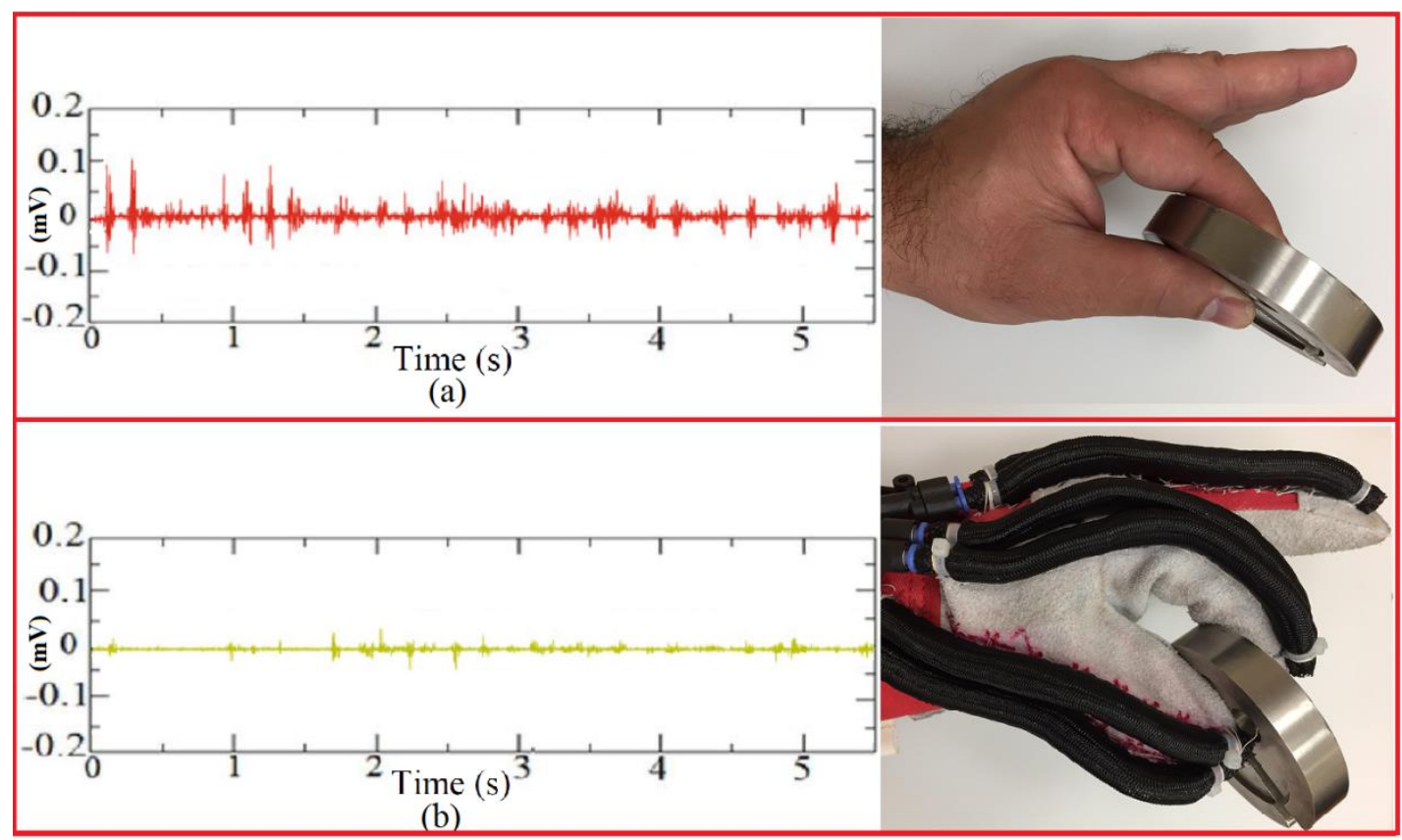

Fig. 16. EMG signals of pinching load.

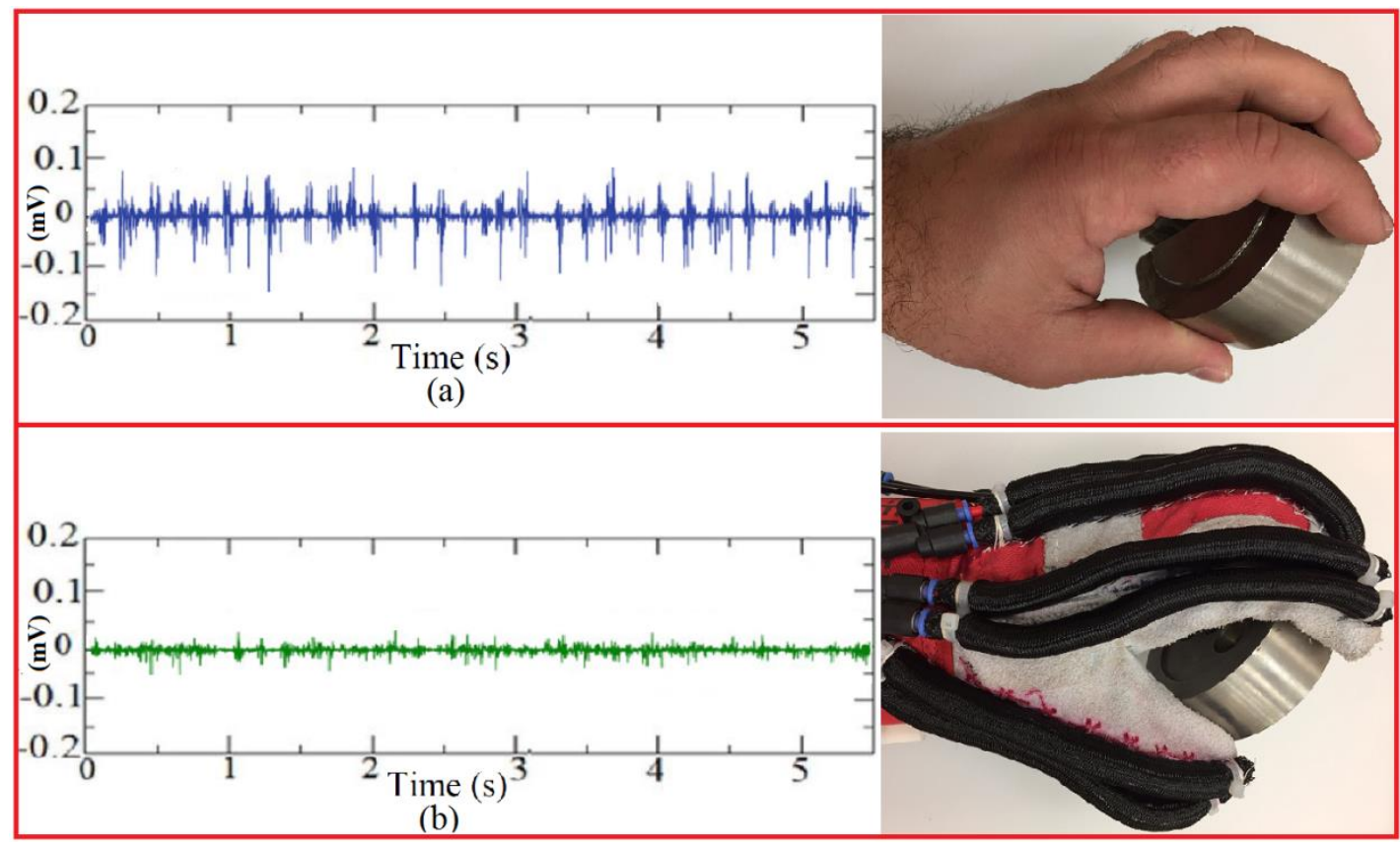

Fig. 17. EMG signals of grasping load. 
From the experimental results above it can be seen that for both grasps the peak voltage measured by the EMG sensors was less when the glove was being used. This indicates that, as would be expected, the user's muscles are required to be less active during the grasps when the glove is used, thus proving the force augmentation capacity of the system. The reduction in required muscle activity would in turn lead to reduced muscle fatigue for the user during extended grasping.

\section{Conclusions}

Human assistance innovation is essential in an increasingly aged society and one technology that may be applicable is exoskeletons. However, traditional rigid exoskeletons have many drawbacks. This paper has introduced a novel bending pneumatic muscle which can be used to develop soft exoskeletons.

The paper has described the construction of a bending pneumatic muscle which is based on an extending McKibben muscle. By reinforcing one side of the muscle to prevent extension a bending motion is produced when the actuator is pressurised. The paper has experimentally assessed the performance of the novel actuator and an output force mathematical model for the proposed actuator has been developed. This model relies on upon the geometrical parameters of the extending bending pneumatic muscle to determine the output force as a function of the input pressure. The model has been verified against experimental results for a range of actuator sizes.

To demonstrate the new actuators a soft exoskeleton glove has been produced. Extensor Bending Pneumatic Artificial Muscles were attached to the rear of each finger of the glove. Then pressurized actuators bend and apply forces to the rear of the fingers causing the finger joints to flex. By using EMG sensors to monitor a user's muscle activity, it has been shown that the glove reduces the amount of muscle effort needed to perform a number of simple grasps.

Future work will seek to improve the mathematical model further by considering other losses such as rubber bladder impedance, the friction between the bladder and the braided sleeve and the friction between the fibre threads in the braid. This will be done to enhance the mathematical model and decrease the average percentage error.

The glove described in this paper has only used open loop control. Future work will develop a new version of the exoskeleton which will include sensors such as bending angle sensors, tactile sensors and pressure sensors to capture the hand movement and create a feedback controller for the exoskeleton.

\section{Acknowledgment}

The authors would like to thank the ministry of higher education/Iraq, University of Basrah, computer engineering department for providing scholarship support to the first author of this paper.

\section{References}

[1] R. Gopura, D. Bandara, K. Kiguchi, and G. Mann, "Developments in hardware systems of active upper-limb exoskeleton robots: A review," Robotics and Autonomous Systems, vol. 75, pp. 203-220, 2016.

[2] P. K. Jamwal, S. Hussain, and S. Q. Xie, "Review on design and control aspects of ankle rehabilitation robots," Disability and Rehabilitation: Assistive Technology, vol. 10, pp. 93-101, 2015.

[3] H. In, B. B. Kang, M. Sin, and K.-J. Cho, "Exo-Glove: a wearable robot for the hand with a soft tendon routing system," IEEE Robotics \& Automation Magazine, vol. 22, pp. 97-105, 2015.

[4] M. Cempini, M. Cortese, and N. Vitiello, "A powered finger-thumb wearable hand exoskeleton with self-aligning joint axes," IEEE/ASME Transactions on Mechatronics, vol. 20, pp. 705-716, 2015.

[5] K. Makino, K. Aoki, M. Nakamura, H. Omori, Y. Hanagata, S. Ueda, et al., "Automatic rehabilitation of the Extension Lag using the developed knee assistive instrument," in 9th International Conference on Human System Interactions (HSI), 2016 2016, pp. 386-392.

[6] H. Al-Fahaam, S. Davis, and S. Nefti-Meziani, "Power assistive and rehabilitation wearable robot based on pneumatic soft actuators," in 21st International Conference on Methods and Models in Automation and Robotics (MMAR), 2016 2016, pp. 472-477.

[7] H. Al-Fahaam, S. Davis, and S. Nefti-Meziani, "Wrist rehabilitation exoskeleton robot based on pneumatic soft actuators," in Students on Applied Engineering (ISCAE), International Conference for, 2016, pp. 491-496. 
[8] E. Rocon and J. L. Pons, Exoskeletons in rehabilitation robotics: Tremor suppression vol. 69: Springer, 2011.

[9] P. Polygerinos, K. C. Galloway, S. Sanan, M. Herman, and C. J. Walsh, "EMG controlled soft robotic glove for assistance during activities of daily living," in Rehabilitation Robotics (ICORR), 2015 IEEE International Conference on, 2015, pp. 55-60.

[10] P. Polygerinos, Z. Wang, K. C. Galloway, R. J. Wood, and C. J. Walsh, "Soft robotic glove for combined assistance and at-home rehabilitation," Robotics and Autonomous Systems, vol. 73, pp. 135-143, 2015.

[11] T. Noritsugu, M. Takaiwa, and D. Sasaki, "Power assist wear driven with pneumatic rubber artificial muscles," in Mechatronics and Machine Vision in Practice, 2008. M2VIP 2008. 15th International Conference on, 2008, pp. 539-544.

[12] K. Toya, T. Miyagawa, and Y. Kubota, "Power-assist glove operated by predicting the grasping mode," Journal of System Design and Dynamics, vol. 5, pp. 94-108, 2011.

[13] Y. Kadowaki, T. Noritsugu, M. Takaiwa, D. Sasaki, and M. Kato, "Development of soft powerassist glove and control based on human intent," Journal of Robotics and Mechatronics, vol. 23, p. 281, 2011.

[14] D. Sasaki, T. Noritsugu, M. Takaiwa, and H. Konishi, "Control Method Based on EMG for Power Assist Glove Using Self-Organizing Maps," Journal ref: International Journal of Automation Technology, vol. 8, pp. 177-185, 2014.

[15] P. Polygerinos, S. Lyne, Z. Wang, L. F. Nicolini, B. Mosadegh, G. M. Whitesides, et al., "Towards a soft pneumatic glove for hand rehabilitation," in Intelligent Robots and Systems (IROS), 2013 IEEE/RSJ International Conference on, 2013, pp. 1512-1517.

[16] H. K. Yap, J. H. Lim, F. Nasrallah, J. C. Goh, and R. C. Yeow, "A soft exoskeleton for hand assistive and rehabilitation application using pneumatic actuators with variable stiffness," in Robotics and Automation (ICRA), 2015 IEEE International Conference on, 2015, pp. 49674972.

[17] J. E. Takosoglu, P. A. Laski, S. Blasiak, G. Bracha, and D. Pietrala, "Determining the static characteristics of pneumatic muscles," Measurement and Control, vol. 49, pp. 62-71, 2016.

[18] G. Andrikopoulos, G. Nikolakopoulos, and S. Manesis, "Pneumatic artificial muscles: A switching Model Predictive Control approach," Control Engineering Practice, vol. 21, pp. 1653-1664, 2013.

[19] G. Andrikopoulos, G. Nikolakopoulos, and S. Manesis, "Design and development of an exoskeletal wrist prototype via pneumatic artificial muscles," Meccanica, vol. 50, pp. 27092730, 2015.

[20] D. Gryparis, G. Andrikopoulos, and S. Manesis, "Parallel robotic manipulation via Pneumatic Artificial Muscles," in 11th International Conference on Informatics in Control, Automation and Robotics (ICINCO), 2014 2014, pp. 29-36.

[21] N. Tsagarakis and D. G. Caldwell, "Improved modelling and assessment of pneumatic muscle actuators," in IEEE International Conference on Robotics and Automation, 2000. Proceedings. ICRA'00. , 2000, pp. 3641-3646.

[22] J. Burgner-Kahrs, D. C. Rucker, and H. Choset, "Continuum robots for medical applications: A survey," IEEE Transactions on Robotics, vol. 31, pp. 1261-1280, 2015.

[23] K. Kadota, M. Akai, K. Kawashima, and T. Kagawa, "Development of Power-Assist Robot Arm using pneumatic rubbermuscles with a balloon sensor," in Robot and Human Interactive Communication, 2009. RO-MAN 2009. The 18th IEEE International Symposium on, 2009, pp. 546-551.

[24] T. E. Pillsbury, Q. Guan, and N. M. Wereley, "Comparison of contractile and extensile pneumatic artificial muscles," in IEEE International Conference on Advanced Intelligent Mechatronics (AIM), 2016 2016, pp. 94-99.

[25] B. Tondu, "Modelling of the McKibben artificial muscle: A review," Journal of Intelligent Material Systems and Structures, vol. 23, pp. 225-253, 2012. 
[26] G. Andrikopoulos, G. Nikolakopoulos, and S. Manesis, "A survey on applications of pneumatic artificial muscles," in 19th Mediterranean Conference on Control \& Automation (MED), 2011 2011, pp. 1439-1446.

[27] D. G. Caldwell, G. A. Medrano-Cerda, and M. Goodwin, "Control of pneumatic muscle actuators," IEEE control systems, vol. 15, pp. 40-48, 1995.

[28] F. Daerden and D. Lefeber, "The concept and design of pleated pneumatic artificial muscles," International Journal of Fluid Power, vol. 2, pp. 41-50, 2001.

[29] C.-P. Chou and B. Hannaford, "Measurement and modeling of McKibben pneumatic artificial muscles," IEEE Transactions on robotics and automation, vol. 12, pp. 90-102, 1996.

[30] B. Tondu and P. Lopez, "Modeling and control of McKibben artificial muscle robot actuators," IEEE control systems, vol. 20, pp. 15-38, 2000.

[31] H. M. Paynter, "Thermodynamic treatment of tug-\&-twist technology: Part 1. Thermodynamic tugger design," in Proceedings, Japan-USA Symposium on Flexible Automation, Boston, MA, 1996, pp. 111-117.

[32] S. Davis, N. Tsagarakis, J. Canderle, and D. G. Caldwell, "Enhanced modelling and performance in braided pneumatic muscle actuators," The International Journal of Robotics Research, vol. 22, pp. 213-227, 2003.

[33] M. Doumit, A. Fahim, and M. Munro, "Analytical modeling and experimental validation of the braided pneumatic muscle," IEEE transactions on robotics, vol. 25, pp. 1282-1291, 2009.

[34] J. Serres, D. Reynolds, C. Phillips, D. Rogers, and D. Repperger, "Characterisation of a pneumatic muscle test station with two dynamic plants in cascade," Computer methods in biomechanics and biomedical engineering, vol. 13, pp. 11-18, 2010.

[35] K. C. Wickramatunge and T. Leephakpreeda, "Study on mechanical behaviors of pneumatic artificial muscle," International Journal of Engineering Science, vol. 48, pp. 188-198, 2010.

[36] J.-F. Zhang, C.-J. Yang, Y. Chen, Y. Zhang, and Y.-M. Dong, "Modeling and control of a curved pneumatic muscle actuator for wearable elbow exoskeleton," Mechatronics, vol. 18, pp. 448-457, 2008.

[37] A. Al-Ibadi, S. Nefti-Meziani, and S. Davis, "Valuable experimental model of contraction pneumatic muscle actuator," in 21st International Conference on Methods and Models in Automation and Robotics (MMAR), 2016 2016, pp. 744-749.

[38] T. Kiminori, T. Miyagawa, and Y. Kubota, "Power-assist glove operated by predicting the grasping mode," Journal of System Design and Dynamics, vol. 5, pp. 94-108, 2011. 